Check for updates

Cite this: J. Anal. At. Spectrom., 2018 33,1284

Received 7th June 2018

Accepted 29th June 2018

DOI: $10.1039 / \mathrm{c} 8 \mathrm{ja} 00192 \mathrm{~h}$

rsc.li/jaas

\title{
Sample preparation strategies for petroleum coke digestion and further cerium and lanthanum determination by DSN-ICP-OES
}

\author{
J. P. Souza, K. Kellermann, M. S. Camargo, D. P. Moraes, (DD D. Pozebon \\ and J. S. F. Pereira (D) *
}

\begin{abstract}
The determination of cerium and lanthanum in petroleum coke samples by inductively coupled plasma optical emission spectrometry coupled to a desolvating nebulizer system (DSN-ICP-OES) is proposed for the first time after studying the following sample preparation methods: microwave-assisted digestion (MW-AD) in medium and high pressure systems and microwave-induced combustion (MIC). MW-AD in a medium pressure system was not suitable for digestion of petroleum coke even when using a low sample mass (up to $100 \mathrm{mg}$ ). Both MIC and MW-AD in a high pressure system were considered suitable for petroleum coke digestion, but MIC was preferable in view of the possibility of using diluted acid (2.5 mol L $\mathrm{m}^{-1} \mathrm{HNO}_{3}$ ) to digest $400 \mathrm{mg}$ of sample and obtaining lower residual carbon content (RCC) values (below $0.1 \%$ instead of approximately $25 \%$ for $M W-A D$ ). The use of $M W-A D$ required a dilution step (with a factor of four) to minimize the interference in analyte determination by DSN-ICP-OES. Accuracy was evaluated by comparing the results with those obtained by neutron activation analysis (an agreement of more than $94 \%$ was obtained) and also analyzing spiked samples (recoveries in the range of 88 to $102 \%$ were achieved).
\end{abstract}

\section{Introduction}

Nowadays, fluid catalytic cracking (FCC) plays a key role in crude oil refining in converting higher molecular weight hydrocarbons to lighter ones by using catalysts (normally zeolites) ${ }^{1}$ that may contain lanthanum and cerium. These elements act as a "bridge" to stabilize the zeolite structure when the catalyst is exposed to high temperature, thus improving the thermal and hydrothermal stability. ${ }^{\mathbf{1 , 2}}$ Therefore, the determination of $\mathrm{Ce}$ and $\mathrm{La}$ in petroleum coke is of great importance because (i) the loss of Ce and La from the catalyst to the crude oil derivatives may cause destabilization of the catalyst and (ii) the presence of Ce and La may contaminate the petroleum coke that is normally required at high purity, since the heavy oil streams produced in FCC can be used as feedstock in the thermal processing units as delayed coke. ${ }^{1,2}$ Therefore, determining $\mathrm{Ce}$ and $\mathrm{La}$ in petroleum coke is a way of identifying catalyst degradation during crude oil processing and is also important for monitoring the presence of these contaminants.

Despite the relevance of Ce and La determination in crude oil derivatives, such as petroleum coke, no studies were found in the literature. One possible reason is the complexity and stability of the petroleum coke matrix combined with the difficulties in determining rare earth elements (REE), including Ce

Instituto de Química, Universidade Federal do Rio Grande do Sul, 91501-970, Porto Alegre, RS, Brazil. E-mail: julianasfp@ufrgs.br; Tel: +55 5133087195 and La. Petroleum coke has a high carbon content $(95 \%$ or higher) with high levels of condensed aromatic rings, leading to the complex and stable matrix. ${ }^{3}$

Based on this, the development of sample preparation methods compatible with the inductively coupled plasma optical emission (ICP-OES) technique is important in order to minimize interference for the determination of Ce and La and to provide reliable results. This technique could be improved with the use of a desolvation system for sample nebulization, achieving better sensitivity and lower limits of quantification (LOQs). Most studies published in the literature for the digestion of petroleum coke discuss further $\mathrm{Cl}, \mathrm{S}, \mathrm{Ni}$, and $\mathrm{V}$ determination. ${ }^{4-12}$ In these studies, sample preparation based on wet digestion with conductive heating, ${ }^{\mathbf{9}, 13}$ microwave-assisted digestion (MW-AD), ${ }^{10-12,14,15}$ dry ashing $^{8}$ and microwaveinduced combustion (MIC) $)^{5-8}$ are reported. The wet digestion method was applied to petroleum coke digestion for further $\mathrm{S}$, $\mathrm{P}^{9}$ and $\mathrm{As}^{13}$ determination. In these cases, digestion was performed in open vessels using several successive steps for sample decomposition and addition of several reagents $\left(\mathrm{KMnO}_{4}\right.$, $\mathrm{K}_{2} \mathrm{Cr}_{2} \mathrm{O}_{7}, \mathrm{HNO}_{3}, \mathrm{HClO}_{4}, \mathrm{Br}_{2}, \mathrm{HF}, \mathrm{HCl}$ and $\mathrm{H}_{2} \mathrm{SO}_{4}$ ). In most of the studies incomplete digestion was observed and solutions with high residual acidity were achieved.

Consequently, MW-AD ${ }^{10-12,14,15}$ and combustion methods ${ }^{8-12}$ have been proposed for the digestion of matrices with a high carbon content that are difficult to dissolve, such as petroleum coke. However, MW-AD could be unsuitable for digestion of 


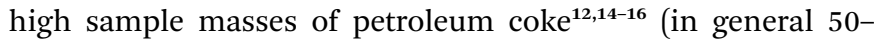
$100 \mathrm{mg}$ of sample were used) and solid residues sometimes remained in the final solution. This necessitates high amounts of inorganic acids and leads to the production of high volumes of hazardous waste. It is important to highlight that in some cases HF was used, and this reagent can not be supported by some analytical techniques, such as ICP-MS and ICP-OES. ${ }^{17}$

Only two studies have been published in the literature involving the MIC method and petroleum coke. In these studies, samples were digested with high efficiency for further $\mathrm{Cl}^{5}$ and $\mathrm{S}^{6}$ determination and in both cases, $500 \mathrm{mg}$ of coke was digested and analytes were retained in water ${ }^{5}$ and $0.5 \mathrm{~mol} \mathrm{~L}^{-1}$ $\left(\mathrm{NH}_{4}\right)_{2} \mathrm{CO}_{3} \cdot{ }^{6}$ Despite the importance of $\mathrm{Ce}$ and La determination in petroleum coke, no studies were found in the literature, regardless of the sample preparation method used.

In the present work an evaluation of sample preparation methods for petroleum coke digestion was performed for the first time for subsequent Ce and La determination by ICP-OES using a desolvating nebulizer (DSN). Sample preparation methods were investigated thoroughly in order to develop high efficiency strategies (evaluated by measuring the RCC in final digests) for petroleum coke digestion resulting in final solutions that are compatible with DSN-ICP-OES. As no certified reference material was available for $\mathrm{Ce}$ and $\mathrm{La}$ in petroleum coke, accuracy was evaluated by comparing results with those obtained by neutron activation analysis (NAA) and analyzing spiked samples.

\section{Experimental}

\subsection{Instrumentation}

A microwave sample preparation system (Multiwave PRO, Anton Paar, Austria) with software version 2.42.7406.8 was used to digest petroleum coke samples by microwave-assisted digestion (MW-AD) under high pressure and by microwave-induced combustion (MIC). The system was equipped with up to eight high-pressure quartz vessels (rotor type 8NXQ 80) under a maximum pressure, temperature and internal volume of 80 bar, $280{ }^{\circ} \mathrm{C}$ and $80 \mathrm{~mL}$, respectively. For MIC digestion, commercial combustion quartz holders (Anton Paar, Austria) were used. Petroleum coke samples were also digested by MW$\mathrm{AD}$ under medium pressure using a SpeedWave Four system (Berghof, Germany), equipped with closed vessels of TFM ${ }^{\circledR}$ PTFE (DAP 30), with a maximum capacity, temperature, pressure and power of $30 \mathrm{~mL}, 230{ }^{\circ} \mathrm{C}, 35$ bar and $1450 \mathrm{~W}$, respectively.

The efficiency of digestion was evaluated by determining the amount of dissolved carbon in the final digests, expressed as the residual carbon content (RCC) relative to the total carbon content in the solid coke samples. The amount of dissolved carbon was determined using a multi N/C 2100S (Analytik Jena, Germany) equipped with an infrared detector using a Focus Radiation System (NDIR, Analytik Jena, Germany) and autosampler AS60. Sample solutions were first acidified with $0.01 \mathrm{~mol} \mathrm{~L}^{-1} \mathrm{HCl}$ and then purged with oxygen for $3 \mathrm{~min}$ in order to remove the volatile carbon compounds before combustion at up to $850{ }^{\circ} \mathrm{C}$ in a furnace. An infrared detector was used for the precise detection of $\mathrm{CO}_{2}$.

Cerium and La determination was carried out using an inductively coupled plasma optical emission spectrometer (model Optima 2000 DV, Perkin Elmer, USA) equipped with a concentric perfluoroalkoxide (PFA) micronebulizer, coupled to an aerosol desolvation system (APEX-Q ${ }^{\circledR}$ model, Elemental Scientific, USA). In the APEX-Q system, the cyclonic spray chamber was maintained at $140{ }^{\circ} \mathrm{C}$, while the condensers were kept at room temperature (condenser 1 ) and at $2{ }^{\circ} \mathrm{C}$ (condenser 2 ). Argon with purity higher than $99.996 \%$ (Oxisul, Brazil) was used as the plasma, nebulizer and auxiliary gas. The experimental conditions used in the DSN-ICP-OES for Ce and La determination are summarized in Table 1.

An analytical balance (AY220, Shimadzu, Brazil) with a resolution and maximum capacity of $0.0001 \mathrm{~g}$ and $220 \mathrm{~g}$, respectively, was used to weigh the petroleum coke samples prior to digestion. The other equipment used were an oven (42LT, Nova Etica, Brazil) to dry samples prior to digestion and a mill (A11, basic S32, IKA, Brazil) to homogenize the samples.

\subsection{Samples, reagents and standards}

Ten samples of petroleum coke, denoted 1 to 10 , were dried in an oven at $60{ }^{\circ} \mathrm{C}$ and homogenized in a cryogenic mill to achieve a particle size $\leq 100 \mu \mathrm{m}$.

All solutions used in this study were prepared with ultrapure water which was purified by distillation and deionization, and then further purified in a Milli-Q system (Millipore Corp., USA) with a minimum resistivity of $18.2 \mathrm{M} \Omega \mathrm{cm}$ at $25^{\circ} \mathrm{C}$. Concentrated nitric acid (Merck, Germany) was distilled in a subboiling system (Model Distillacid BSB-939-IR, Berghof, Germany) and was used for petroleum coke digestion by MIC and MW-AD. Hydrogen peroxide (30\%, Merck, Germany) was used as an auxiliary reagent.

A $6 \mathrm{~mol} \mathrm{~L}^{-1}$ ammonium nitrate solution was prepared from the solid reagent (Merck, Germany) and was used as an initiator in the MIC procedure. Oxygen (99.999\% purity, Air Liquide) was used in the digestion by MIC.

Table 1 Operational conditions used for $\mathrm{Ce}$ and La determination by DSN-ICP-OES

\begin{tabular}{ll}
\hline Parameter & Condition \\
\hline Nebulizer system & APEX-Q \\
Sample flow rate, $\mathrm{mL} \mathrm{min}^{-1}$ & 0.70 \\
Spray chamber & Cyclonic \\
Heating/cooling temperatures, ${ }^{\circ} \mathrm{C}$ & $140 /$ room temperature and 2 \\
Nebulizer gas flow rate, $\mathrm{L} \mathrm{min}^{-1}$ & $0.55-0.85$ \\
Plasma gas flow rate, $\mathrm{L} \mathrm{min}^{-1}$ & 15 \\
Auxiliary gas flow rate, $\mathrm{L} \mathrm{min}^{-1}$ & 0.2 \\
RF Power, W & $1200-1500$ \\
Replicates & 3 \\
View & $\mathrm{Axial}$ \\
Wavelength, nm & $\mathrm{Ce}(\mathrm{II}) 413.764$ \\
& $\mathrm{La}(\mathrm{II}) 398.852$ \\
& ${ }^{a} \mathrm{Yb}(\mathrm{II}) 369.419$ \\
&
\end{tabular}


The calibration curve used for the carbon measurements was prepared by dissolving potassium hydrogen phthalate $\left(\mathrm{C}_{8} \mathrm{H}_{5} \mathrm{O}_{4} \mathrm{~K}\right)$ (Merck, Germany) in water to prepare a stock solution containing $10 \mathrm{~g} \mathrm{~L}^{-1}$ carbon. This was used to prepare standards by sequential dilution.

A multi-element stock solution containing $10 \mathrm{mg} \mathrm{L}^{-1} \mathrm{Ce}$ and La was used to prepare analytical standards by sequential dilution in $5 \%(\mathrm{v} / \mathrm{v}) \mathrm{HNO}_{3}$ (Merck) solution with concentrations ranging from 1 to $100 \mu \mathrm{g} \mathrm{L} \mathrm{L}^{-1}$. When the internal standard was used, suitable volumes of the stock solution containing $10 \mathrm{mg} \mathrm{L}^{-1} \mathrm{Yb}$ (Merck, Germany) were added to the calibration solutions and samples prior to analysis.

\subsection{Sample preparation strategies for petroleum coke digestion}

For sample digestion by $\mathrm{MW}-\mathrm{AD}$ in a medium pressure system, $6 \mathrm{~mL}$ of $\mathrm{HNO}_{3}$ and $2 \mathrm{~mL}$ of $\mathrm{H}_{2} \mathrm{O}_{2}$ were added to PTFE vessels containing $100 \mathrm{mg}$ of sample. The sample was then heated as follows: (i) $15 \mathrm{~min}$ at $145{ }^{\circ} \mathrm{C}$ (ramp of $2 \mathrm{~min}$ ) and a power of $80 \%$, (ii) $10 \mathrm{~min}$ at $170{ }^{\circ} \mathrm{C}$ (ramp of $5 \mathrm{~min}$ ) and a power of $90 \%$ and (iii) $20 \mathrm{~min}$ at $220{ }^{\circ} \mathrm{C}$ (ramp of $2 \mathrm{~min}$ ) and a power of $90 \%$, which is a modification of the program recommended by the manufacturer (with longer ramps and hold times). The power settings for this instrument are set as a percentage of the maximum power, which is $1450 \mathrm{~W}(100 \%$ microwave power). After cooling, the solutions were diluted to $25 \mathrm{~mL}$ with ultrapure water.

For MW-AD in a high pressure system (up to 80 bar), the samples (i.e., $375 \mathrm{mg}$ ) were transferred to quartz vessels. After the addition of $6 \mathrm{~mL} \mathrm{HNO}_{3}$, the vessels were closed and fixed to the rotor, which was covered with a lid and then placed in the cavity of the microwave oven. The heating program used was $900 \mathrm{~W}$ for $40 \mathrm{~min}$ (ramp of $15 \mathrm{~min}$ ) and $20 \mathrm{~min}$ at $0 \mathrm{~W}$ for cooling. During the digestion, the maximum pressure, rate of increase of pressure and temperature were set at 80 bar, 0.8 bar $\mathrm{s}^{-1}$ and $280{ }^{\circ} \mathrm{C}$, respectively. After cooling, solutions were diluted to $25 \mathrm{~mL}$ with ultrapure water. Hydrogen peroxide and $\mathrm{HNO}_{3}$ were also studied for sample digestion.

For MIC, petroleum coke samples (i.e., $400 \mathrm{mg}$ ) were pressed into pellets, weighed and placed on the small disk of filter paper positioned at the base of the quartz holder device. Then, $50 \mu \mathrm{L}$ of a $6 \mathrm{~mol} \mathrm{~L}^{-1} \mathrm{NH}_{4} \mathrm{NO}_{3}$ solution was added to the filter paper. The quartz holder device containing the sample was placed into the quartz vessel previously filled with $6 \mathrm{~mL}$ of absorption solution $\left(\mathrm{HNO}_{3}\right)$. The vessels were closed, fixed to the rotor and placed in the cavity of the microwave oven. The vessels were pressurized with 20 bar of oxygen for 30 s. They were then heated at $1200 \mathrm{~W}$ for $30 \mathrm{~s}$ and a maximum power of $1400 \mathrm{~W}$ for

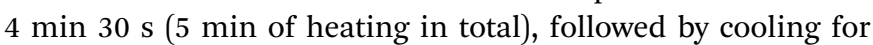
$20 \mathrm{~min}$ at $0 \mathrm{~W}$. After cooling, the rotor was removed and the pressure in the vessels was released. The vessels were opened and the resulting solutions were diluted with ultrapure water to $25 \mathrm{~mL}$.

A summary of the sample preparation strategies used for petroleum coke digestion is shown in Fig. 1.

The accuracy of the sample preparation methods was evaluated by analyte recovery tests. Three concentration levels were evaluated for each analyte and tests were performed for $n=5$. For MW-AD, a suitable volume of the standard reference solution (10 $\mathrm{mg} \mathrm{L}^{-1} \mathrm{Ce}$ and $\mathrm{La}$ ) was added to the sample in the quartz vessel prior to digestion. Similarly, for MIC digestion, the standard reference solution was added to the sample pellet prior to digestion. In both cases, the same procedure was used for the spiked sample as for the sample digestion. Due to the lack of certified reference materials with a similar matrix composition that contain certified values of Ce and La, sample "1" was analyzed by neutron activation analysis (NAA, Comissão

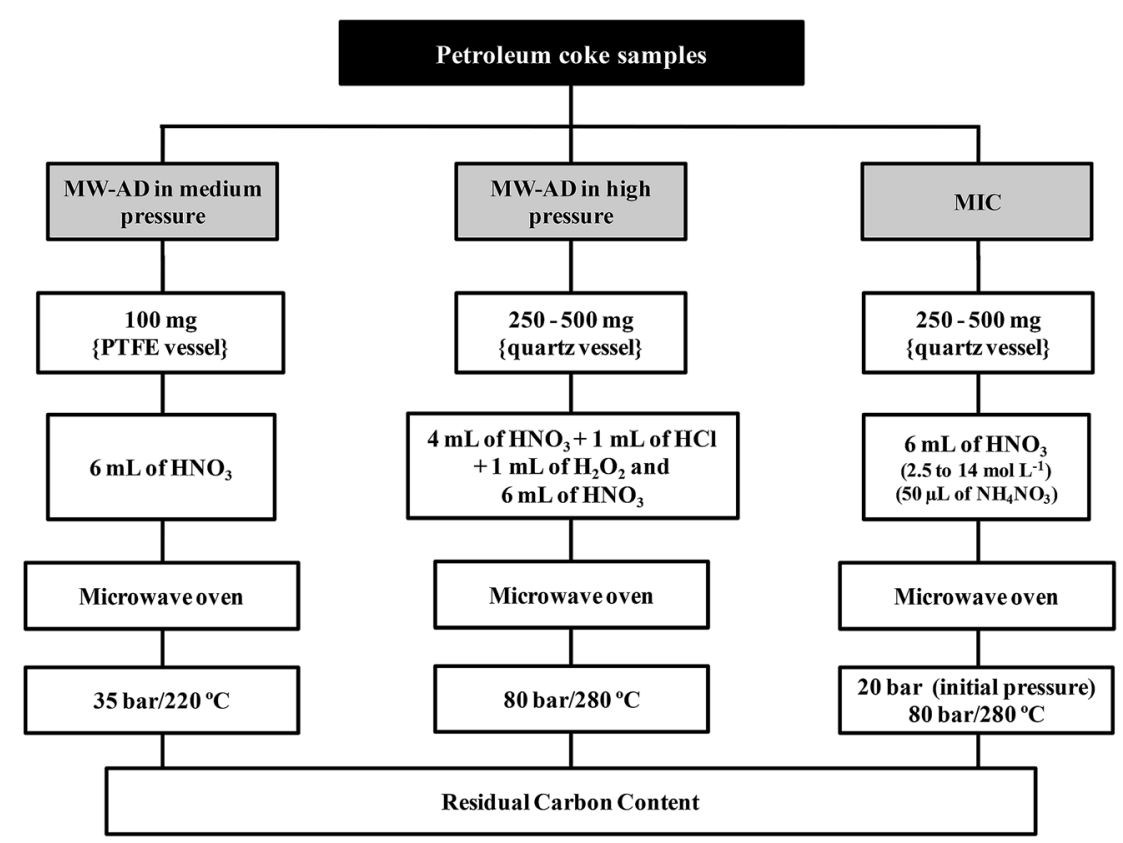

Fig. 1 Summary of sample preparation strategies used for petroleum coke digestion. 
Nacional de Energia Nuclear, Instituto de Pesquisas Energéticas e Nucleares, IPEN, São Paulo, Brazil) using a neutron flow of $10^{12} \mathrm{n} \mathrm{cm}^{-2} \mathrm{~s}^{-1}$. Before the half-life of each element was reached, gamma activity measurements were performed in a hyperpure Ge detector (GX 2020, Canberra) linked to a spectrometer. The radioisotopes measured, energy applied and halflife were ${ }^{141} \mathrm{Ce}(145.4 \mathrm{keV}, 80 \mathrm{~h})$ and ${ }^{140} \mathrm{La}(1596.20 \mathrm{keV}, 8 \mathrm{~h})$.

\subsection{Cerium and lanthanum determination by DSN-ICP-OES}

In order to optimize the conditions for DSN-ICP-OES, the nebulizer gas flow rate and the radiofrequency (RF) power were investigated for the nebulizer with aerosol desolvation. The sample flow rate was fixed according to the manufacturer's recommendations and the nebulizer gas flow rate was varied in increments of $0.05 \mathrm{~L} \mathrm{~min}^{-1}$ maintaining a $\mathrm{RF}$ power of $1300 \mathrm{~W}$. When the RF power was studied, the nebulizer gas flow rate was fixed and increments of $50 \mathrm{~W}$ were used to measure the signals for each analyte. The use of an internal standard was investigated for DSN-ICP-OES measurements to minimize interferences.

\section{Results and discussion}

\subsection{Evaluation of sample preparation strategies for petroleum coke digestion}

Sample preparation methods based on microwave radiation were studied to investigate their applicability in petroleum coke digestion and the suitability of the final solutions for subsequent $\mathrm{Ce}$ and La determination by DSN-ICP-OES. Petroleum coke sample " 1 " was used to optimize all sample preparation methods evaluated in this study.

3.1.1. Microwave-assisted digestion in a medium pressure system. The microwave heating program recommended by the manufacturer for crude oil digestion was initially used. The microwave heating program was as follows for $100 \mathrm{mg}$ of sample with $6 \mathrm{~mL}$ of concentrated $\mathrm{HNO}_{3}$ and $2 \mathrm{~mL}$ of $\mathrm{H}_{2} \mathrm{O}_{2}$ : (i) $145{ }^{\circ} \mathrm{C}$ for $10 \mathrm{~min}$ (ramp of $2 \mathrm{~min}$ ) at $70 \%$ power, (ii) $170{ }^{\circ} \mathrm{C}$ for $5 \mathrm{~min}$ (ramp of $5 \mathrm{~min}$ ) at $80 \%$ power and (iii) $200{ }^{\circ} \mathrm{C}$ for $20 \mathrm{~min}$ (ramp of $2 \mathrm{~min}$ ) at $90 \%$ power. The final solutions contained large quantities of solid residues, and in order to improve digestion efficiency, the hold time of the first and second steps was increased to 15 and $10 \mathrm{~min}$, respectively. However, solid residues remained in the solution. A new digestion procedure was performed where the power applied in the three heating steps was increased to 80,90 and $90 \%$, respectively, and the maximum temperature of the last step was increased from 200 to $220{ }^{\circ} \mathrm{C}$ (the sample mass and volume of the reagents used were kept the same). Under these conditions, the digestion improved considerably, although a solid residue remained. Wasilewska et al. ${ }^{18}$ confirmed that a temperature of $220{ }^{\circ} \mathrm{C}$ is suitable for the oxidation of organic matter, obtaining low RCC for several types of samples and that the efficiency of MW-AD is not dependent on the equipment used. This is in agreement with the observations in this study, and the presence of solid residues in the final solutions in similar equipment has also been reported by other authors, in which maximum temperatures of 180 (ref. 14) and $200{ }^{\circ} \mathrm{C}$ (ref. 16) were used for the digestion of petroleum coke and coal coke, respectively.

When $200 \mathrm{mg}$ of sample were digested (other parameters were kept the same), the bottle cap seal broke due to the excessive pressure reached during the microwave heating procedure. This was also observed by others authors, when a sample mass greater than $50 \mathrm{mg}$ was digested by MW-AD. ${ }^{12}$ Due to these safety issues and the impossibility of digesting samples weighing more than $100 \mathrm{mg}$, this system was not used in further experiments.

3.1.2. Microwave-assisted digestion in a high pressure system. The sample mass $(250 \mathrm{mg}$ ) and heating program recommended by the manufacturer were initially used for the digestion of petroleum coke. The microwave heating program used was as follows: (i) $900 \mathrm{~W}$ for $40 \mathrm{~min}$ (ramp of $15 \mathrm{~min}$ ) and (ii) cooling for $20 \mathrm{~min}$ at $0 \mathrm{~W}$. The maximum pressure rate was 0.3 bar s${ }^{-1}$ with a mixture containing $4 \mathrm{~mL}$ of $\mathrm{HNO}_{3}, 1 \mathrm{~mL}$ of $\mathrm{HCl}$ and $1 \mathrm{~mL}$ of $\mathrm{H}_{2} \mathrm{O}_{2}$ (the maximum pressure and temperature were set at 80 bar and $280{ }^{\circ} \mathrm{C}$, respectively).

This microwave heating procedure resulted in final solutions that were clear and without solid residues. The RCC was determined and was approximately $8.8 \%$. Since the RCC was not significantly high, larger amounts of petroleum coke (500 $\mathrm{mg}$ ) were digested under the same conditions. However, for a $500 \mathrm{mg}$ sample, incomplete digestion occurred and solid residues remained in the solution. In order to improve sample digestion, the pressure rate was changed from 0.3 to $0.8 \mathrm{bar} \mathrm{s}^{-1}$, while maintaining the other parameters. This change led to the reduction of solid residues that remained in the digest, possibly because the microwave irradiation was less frequently interrupted during the procedure due to the high pressure limit (the temperature was always below the limit of $280{ }^{\circ} \mathrm{C}$ ). Therefore, for subsequent investigations, the pressure rate was set at 0.8

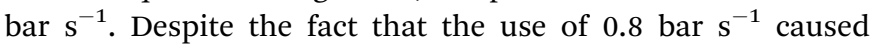
improvements in sample digestion, solid residues were still present in final digests and similar results were obtained using 450 or $400 \mathrm{mg}$ of sample. Therefore, the use of an intermediate sample mass (375 mg) was considered.

When MW-AD was used to digest $375 \mathrm{mg}$ of petroleum coke, the final solution obtained was clear, compared with the solution following the digestion of $500 \mathrm{mg}$, and a RCC of approximately $23 \%$ was obtained. In order to reduce the amounts of the reagents used and, consequently, reduce blank values and possible contamination, an additional experiment was carried out using the same procedure for petroleum coke digestion but using a mixture containing only $6 \mathrm{~mL}$ of concentrated $\mathrm{HNO}_{3}$ (instead of a mixture containing $\mathrm{HNO}_{3}, \mathrm{HCl}$ and $\mathrm{H}_{2} \mathrm{O}_{2}$ ). The removal of $\mathrm{HCl}$ and $\mathrm{H}_{2} \mathrm{O}_{2}$ did not result in any visual differences to the final solutions compared with when $\mathrm{HNO}_{3}$ alone was used. The RCC for the digestion of $375 \mathrm{mg}$ of sample using $\mathrm{HNO}_{3}$ alone was $27.2 \%$, and $23.3 \%$ using a mixture of $\mathrm{HNO}_{3}$, $\mathrm{HCl}$ and $\mathrm{H}_{2} \mathrm{O}_{2}$. This reduction of approximately $4 \%$ was not considered sufficient enough to justify the use of $\mathrm{HCl}$ and $\mathrm{H}_{2} \mathrm{O}_{2}$ in the procedure. Thus, MW-AD in a high pressure system was considered suitable for the digestion of $375 \mathrm{mg}$ of petroleum coke using $6 \mathrm{~mL}$ of concentrated $\mathrm{HNO}_{3}$. This highlights the 
difficulty of digesting petroleum coke, even when using a system under high pressure and temperature.

After establishing better conditions for sample digestion by $\mathrm{MW}-\mathrm{AD}$, analyte recovery tests were performed and recoveries ranging from 88 to 97 and 92 to $98 \%$ were obtained for Ce and La, respectively, using $375 \mathrm{mg}$ of petroleum coke and $6 \mathrm{~mL}$ of concentrated $\mathrm{HNO}_{3}$.

3.1.3. Microwave-induced combustion. The MIC method was investigated as a sample preparation strategy for petroleum coke digestion in view of its high efficiency in decomposing complex samples. ${ }^{19,20}$ Initially, MIC was performed with $500 \mathrm{mg}$ of petroleum coke, an oxygen pressure of $20 \mathrm{bar}$, the addition of $50 \mu \mathrm{L}$ of $6 \mathrm{~mol} \mathrm{~L}^{-1} \mathrm{NH}_{4} \mathrm{NO}_{3}$ for ignition and a 5 min reflux step. Following this the sample was not completely digested (solid residue was observed in the quartz holder). This residue was not attributed to the inorganic fraction of the sample (ash), because the ash content of the petroleum coke samples was lower than $0.1 \%$. This behaviour has not been observed by other authors. ${ }^{5,7,8,10}$

It is notable that in almost all studies related to MIC, the software version was changed to v1.27-Synt to run with a maximum pressure rate of 3 bar $\mathrm{s}^{-1}$. This is important in order to maintain the microwave radiation during the first few seconds when the sample is combusted and the pressure rapidly increases. However, the commercialized equipment (software version 2.42.7406.8) no longer allows modifications to the software version to run digestions. Therefore, the MIC heating program was initialized with $1200 \mathrm{~W}$ (the maximum microwave power permitted by the software) and this power was maintained for the first $30 \mathrm{~s}$ of irradiation the maximum pressure rate allowed is 0.8 bar s$^{-1}$ ). After $30 \mathrm{~s}$, the microwave power was increased to the value established in the microwave heating program $(1400 \mathrm{~W})$. This may explain the different behaviour observed for sample combustion, as in the first moment that microwave radiation is generated, sample combustion begins, and the microwave power is limited to $1200 \mathrm{~W}$.

In order to improve the efficiency of combustion, the $\mathrm{O}_{2}$ pressure was increased to 25 bar, and the digestion was performed using two disks of filter paper, containing $50 \mu \mathrm{L}$ of $6 \mathrm{~mol} \mathrm{~L}{ }^{-1} \mathrm{NH}_{4} \mathrm{NO}_{3}$. After digestion, the amount of residue observed was practically the same. As such, no differences were observed when using one or two disks of filter paper or using 20 or 25 bar of oxygen. In order to obtain efficient digestion, the sample mass was reduced to $400 \mathrm{mg}$ (all other conditions were maintained). After digestion, the amount of solid residue present in the sample quartz holder was significantly reduced, and following the reflux step ( $5 \mathrm{~min}$ ), no apparent residues were observed in the sample holder. Thus, MIC was suitable for the digestion of up to $400 \mathrm{mg}$ of petroleum coke.

After investigating the maximum sample mass, the concentration of the absorbing solution was studied and the results for Ce and La are shown in Fig. 2. The agreement of the results obtained for all absorbing solutions investigated was calculated considering the average of the results obtained by NAA as $100 \%$.

Similar results were obtained for all the absorbing solutions investigated (ANOVA, confidence level of 95\%) and an

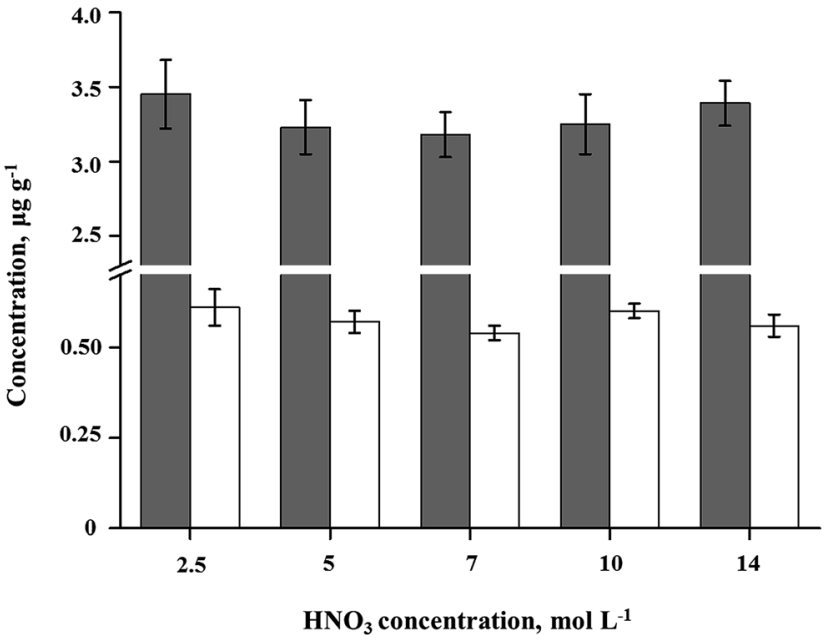

Fig. 2 . Results ( $n=3$ ) obtained for Ce ( $\square$ ) and La ( $\square$ ) varying the concentration of $\mathrm{HNO}_{3}$ used as the absorption solution by MIC.

agreement with results using NAA of more than $90 \%$ was achieved for Ce and La when at least $2.5 \mathrm{~mol} \mathrm{~L}^{-1} \mathrm{HNO}_{3}$ solution was used. The digestion efficiency of the MIC method was evaluated by RCC measurements in the final digests, and it was always below $0.1 \%$, independent of the concentration of the absorbing solution used. Therefore, better conditions for petroleum coke digestion by MIC were obtained using $400 \mathrm{mg}$ of sample, pressurization with 20 bar of oxygen and a solution containing at least $2.5 \mathrm{~mol} \mathrm{~L}^{-1} \mathrm{HNO}_{3}$ as the absorbing solution for analyte retention. The recoveries obtained for $\mathrm{Ce}$ and $\mathrm{La}$ after MIC digestion of spiked samples were in the range of 90 to 98 and 93 to $102 \%$, respectively, and RSD values were lower than $5 \%$ for all analytes using $2.5 \mathrm{~mol} \mathrm{~L}^{-1} \mathrm{HNO}_{3}$ as the absorbing solution for analyte retention.

\subsection{Evaluation of cerium and lanthanum determination by DSN-ICP-OES and internal standardization}

For La and Ce determination using the nebulizer with a desolvation system, the sample flow rate was fixed at $0.7 \mathrm{~mL} \mathrm{~min}^{-1}$ and the nebulizer gas flow rate and RF power of the plasma were investigated in order to improve the sensitivity for analyte determination by ICP-OES. Higher intensity analyte signals were observed when the nebulizer gas flow rate was set at about 0.70-0.75 $\mathrm{L} \mathrm{min}^{-1}$, and for subsequent measurements, 0.75 $\mathrm{L} \min ^{-1}$ was set as the optimum condition. The intensities of the Ce and La signals were similar when increments of $50 \mathrm{~W}$ of RF power were used from 1200 to $1500 \mathrm{~W}$. A RF power of $1300 \mathrm{~W}$ was adopted for subsequent tests, also taking into account the $\mathrm{RF}$ power recommended by the manufacturer of the ICP-OES instrument.

After petroleum coke sample digestion, analytes were determined by DSN-ICP-OES using the conditions previously described. Initially, analyte determination by DSN-ICP-OES in MW-AD digests was performed without pre-dilution, giving 0.23 \pm 0.15 and $2.94 \pm 0.18 \mu \mathrm{g} \mathrm{g}^{-1} \mathrm{Ce}$ and La, respectively. These results correspond to approximately 38 and $84 \%$ of those obtained from the reference method (NAA). Considering that these 
differences could be caused by the RCC in the final solutions, increasing the density and viscosity of the sample solution and consequently affecting nebulization, serial sample dilution was performed prior to analyte measurements $(2,4,6,8$ and 10 times). When a dilution factor greater than 4 was applied, the concentrations of Ce and La were below the LOQs obtained by DSN-ICP-OES. Furthermore, using a dilution factor of at least 4, the Ce and La concentrations obtained by MW-AD were similar ( $t$-test, $95 \%$ confidence level) to those obtained by NAA. The concentrations of $\mathrm{Ce}$ and La obtained in the final solutions by MIC were $0.65 \pm 0.07$ and $3.32 \pm 0.08 \mu \mathrm{g} \mathrm{g}{ }^{-1}$, respectively, corresponding to an agreement of greater than $94 \%$ compared with NAA. Due to the low RCC, no dilution step was used prior to analyte determination.

In order to avoid sample dilution before analyte determination by DSN-ICP-OES when using MW-AD, the use of an internal standard was investigated. $\mathrm{Yb}(369.419 \mathrm{~nm})$ was selected due to its similar behaviour to the analytes in the plasma (an ionization potential of $12.18 \mathrm{eV}$ and similar behaviour when the parameters of the plasma were investigated). The emission line of $\mathrm{Yb}$ of $328.937 \mathrm{~nm}$ was not used due to interference caused by an overlap with the $\mathrm{V}$ emission line (328.939 $\mathrm{nm}$ ).

To investigate the IS, solutions obtained from MIC digestion using $6 \mathrm{~mL}$ of $2.5 \mathrm{~mol} \mathrm{~L}^{-1} \mathrm{HNO}_{3}$ and from MW-AD using $6 \mathrm{~mL}$ of concentrated $\mathrm{HNO}_{3}$ were evaluated. The results obtained when using the IS for Ce $\left(0.65 \pm 0.07 \mu \mathrm{g} \mathrm{g}^{-1}\right)$ and $\mathrm{La}(3.32 \pm$ $0.08 \mu \mathrm{g} \mathrm{g}^{-1}$ ) after MIC digestion were similar to those obtained without using the IS ( $t$-test, 95\% confidence level). However, for MW-AD digests, results using the IS were increased in comparison with those obtained without using the IS, determined in a concentrated solution (without using a pre-dilution step). In this case, Ce and La concentrations in final solutions obtained by MW-AD without pre-dilution were $0.64 \pm 0.05$ and $3.37 \pm 0.27 \mu \mathrm{g} \mathrm{g}^{-1}$, respectively, when using the IS, corresponding to agreements of 107 and $96 \%$ compared with results obtained by NAA (no statistical difference was observed between the results of the $t$-test at the $95 \%$ confidence level). Therefore, the IS was able to minimize the interference observed during analyte determination when MW-AD was used for petroleum coke digestion, avoiding the dilution step and achieving better LOQs. Thus, the IS was used for all measurements.

\subsection{Analytical performance and applications}

The results were compared with those obtained by the reference method (NAA) in order to evaluate the accuracy of all the sample preparation methods investigated since no certified reference materials are available for the same sample matrix containing Ce and La.

The results obtained for NAA for petroleum coke sample " 1 " were $3.52 \pm 0.30(\mathrm{La})$ and $0.60 \pm 0.40(\mathrm{Ce}) \mu \mathrm{g} \mathrm{g}^{-1}$. Agreement values of 94 and 108\% were obtained for La and Ce by MIC, respectively, compared with the values obtained by NAA, and the respective agreement values for MW-AD were approximately 96 and 107\%. From the results obtained for accuracy, both sample preparation methods (MIC and MW-
AD) could be considered suitable for petroleum coke digestion, despite the particularities of each of them. The accuracies and LOQs obtained for both methods investigated are shown in Table 2.

The use of concentrated acids in the MW-AD required the use of an internal standard to minimize interference in the determination step by DSN-ICP-OES if no pre-dilution step was performed. If no internal standard was used, a pre-dilution step of a factor of at least 4 was required resulting in higher LOQs.

The Ce and La concentrations in petroleum coke samples after MIC and MW-AD, determined by DSN-ICP-OES using internal standardization, are shown in Table 3.

Cerium and La were present in low concentrations in the samples investigated. The $\mathrm{La}$ and Ce concentrations in the petroleum coke samples (ratio $\mathrm{La}: \mathrm{Ce}>1$ ) were different to those observed in crude oils ( $\mathrm{La}: \mathrm{Ce}<1$ ) according to the literature. ${ }^{19,21-28}$ It could be hypothesised that the presence of these elements in petroleum coke samples is due to an external effect, such as contamination during refining or by catalyst loss during FCC. The ratio of $\mathrm{La}$ : $\mathrm{Ce}>1$ observed in the major catalysts used in FCC reinforces the hypothesis ${ }^{29-31}$ that their presence is due to the contamination by catalysts during the refining process. Therefore, the catalyst may be lost to the atmosphere, ${ }^{29,32}$ and transferred to the refining products, such as petroleum coke, leading to contamination.

Table 2 Results (and standard deviations) for accuracy evaluation for Ce and La $(n=3)$ obtained after MIC and MW-AD using an internal standard for DSN-ICP-OES measurements

\begin{tabular}{llllll}
\hline \multirow{2}{*}{$\begin{array}{l}\text { Sample } \\
\text { preparation }\end{array}$} & \multicolumn{2}{l}{ Concentration $\left(\mu \mathrm{g} \mathrm{g}^{-1}\right)$} & & \multicolumn{2}{l}{ LOQ $\left(\mu \mathrm{g} \mathrm{g}^{-1}\right)$} \\
\cline { 2 - 3 } \cline { 5 - 6 } & Ce & La & & Ce & La \\
\hline MIC & $0.65 \pm 0.07$ & $3.32 \pm 0.08$ & & 0.096 & 0.014 \\
MW-AD & $0.64 \pm 0.05$ & $3.37 \pm 0.27$ & $0.10\left(0.41^{a}\right)$ & $0.015\left(0.060^{a}\right)$ \\
NAA & $0.60 \pm 0.40$ & $3.52 \pm 0.30$ & & ni & ni
\end{tabular}

${ }^{a}$ Considering the matrix effect when IS was not used (dilution factor of at least 4). ni: not informed.

Table 3 Results and standard deviations, in $\mu \mathrm{g} \mathrm{g}^{-1}$, obtained for Ce and $\mathrm{La}$ in petroleum coke samples by DSN-ICP-OES after performing sample preparation methods $(n=3)$

\begin{tabular}{llllll}
\hline & \multirow{2}{*}{ MIC } & & \multicolumn{2}{l}{ MW-AD } \\
\cline { 2 - 3 } \cline { 5 - 6 } Sample & Ce & La & & Ce & La \\
\hline 1 & $0.65 \pm 0.07$ & $3.32 \pm 0.08$ & $0.64 \pm 0.05$ & $3.37 \pm 0.27$ \\
2 & $<0.096^{a}$ & $0.25 \pm 0.05$ & $<0.10^{a}$ & $0.23 \pm 0.13$ \\
3 & $<0.096^{a}$ & $<0.014^{a}$ & $<0.10^{a}$ & $<0.015^{a}$ \\
4 & $<0.096^{a}$ & $2.37 \pm 0.09$ & $<0.10^{a}$ & $2.35 \pm 0.19$ \\
5 & $<0.096^{a}$ & $<0.014^{a}$ & $<0.10^{a}$ & $<0.015^{a}$ \\
6 & $<0.096^{a}$ & $<0.014^{a}$ & $<0.10^{a}$ & $<0.015^{a}$ \\
7 & $<0.096^{a}$ & $<0.014^{a}$ & $<0.10^{a}$ & $<0.015^{a}$ \\
8 & $<0.096^{a}$ & $<0.014^{a}$ & & $<0.10^{a}$ & $<0.015^{a}$ \\
9 & $<0.096^{a}$ & $<0.014^{a}$ & & $<0.10^{a}$ & $<0.015^{a}$ \\
10 & $<0.096^{a}$ & $<0.014^{a}$ & & $<0.10^{a}$ & $<0.015^{a}$
\end{tabular}

${ }^{a}$ Limits of quantification obtained by DSN-ICP-OES. 


\subsection{Comparison of sample preparation strategies for petroleum coke digestion}

According to the results shown before, MW-AD requires the use of concentrated acids, which could result in poor LOQs if internal standardization is not used (interference was observed when final solutions were directly analyzed without prior dilution). On the other hand, using MIC allowed the use of a solution as dilute as $2.5 \mathrm{~mol} \mathrm{~L}^{-1} \mathrm{HNO}_{3}$ to retain $\mathrm{La}$ and $\mathrm{Ce}$, avoiding the need for sample dilution before analyte determination by DSNICP-OES. The MIC method allowed lower reagent consumption and residue generation (since only $\mathrm{HNO}_{3}$ was used).

The RCC values in the final digests obtained using MIC are notable. As mentioned previously, the RCC values in the digests obtained by MIC were always below $0.1 \%$. Conversely, RCC values were approximately $27 \%$ when MW-AD was used, causing interference during analyte determination when an IS was not used. Therefore, MIC had better digestion efficiency, even in petroleum coke, which is considered a complex material.

Regarding the digestion time and sample throughput, conditions obtained for both MIC and MW-AD methods were similar, and about eight samples could be decomposed in $3 \mathrm{~h}$, including the time for sample manipulation, digestion and cooling, and cleaning of the vessels and devices. Although MIC required more time for sample manipulation (pellet formation, sample insertion into the quartz vessels and pressurization), the length of time for MW-AD was significantly higher, making the total time for both procedures similar. One of the most important factors in REE determination in petroleum coke samples is the low analyte concentration required in this type of matrix, and using diluted solutions for analyte retention is important in order to avoid dilution steps prior to analyte determination by DSN-ICP-OES resulting in better LOQs.

To our knowledge, this is the first application of petroleum coke decomposition using MIC with diluted solutions for subsequent $\mathrm{Ce}$ and La determination. Therefore, the MIC method could be considered a promising method for the digestion of petroleum coke for subsequent $\mathrm{Ce}$ and La determination.

\section{Conclusions}

Sample preparation strategies based on MW-AD in a high pressure system and MIC were suitable for petroleum coke digestion with subsequent $\mathrm{Ce}$ and La determination by DSNICP-OES. Microwave-assisted digestion in a medium pressure system was considered unsuitable due to the low sample mass that could be digested (100 mg).

Both MIC and MW-AD under high pressure could be considered suitable for petroleum coke digestion, despite their particularities. Using MIC, it was possible to obtain a suitable solution, as dilute as $2.5 \mathrm{~mol} \mathrm{~L}{ }^{-1} \mathrm{HNO}_{3}$, for analyte retention, minimizing interference during the determination step. An agreement greater than $94 \%$ was achieved by comparing the results with those obtained by NAA. The proposed MIC method allowed the combustion of $400 \mathrm{mg}$ of sample, and despite the time required for sample manipulation by MIC being higher than that by MW-AD (due to pellet formation, insertion of the sample into the quartz holder and pressurization), the total time for digestion was similar because MIC took only 5 min to achieve complete sample digestion (instead of $55 \mathrm{~min}$ for the heating program using MW-AD).

The carbon concentration in the digests following $\mathrm{MW}-\mathrm{AD}$ was significantly higher (approximately 27\%) than that after MIC (less than $0.1 \%$ ), making it necessary to perform a dilution of a factor of at least four to minimize interference during analyte determination by DSN-ICP-OES. This could lead to poor LOQs and the inability to determine analyte concentrations in the final digests. On the other hand, with using an internal standard, interference could be minimized, and both sample preparation methods could be considered suitable. As concentrated acids were not required, the MIC method can be considered to be in agreement with green chemistry recommendations.

\section{Conflicts of interest}

There are no conflicts to declare.

\section{Acknowledgements}

The authors are grateful to CNPq (482355/2013-1) and FAPERGS (1992-2551/13-9) for supporting this study.

\section{References}

1 R. A. Meyers, Handbook of Petroleum Refining Processes, McGraw-Hill, New York, 1997.

2 R. Sadeghbeigi, Fluid Catalytic Cracking Handbook, Elsevier, 2000.

3 V. V. Speybroeck, D. V. Neck, M. Waroquier, S. Wauters, M. Saeys and G. B. Marin, Int. J. Quantum Chem., 2003, 91, 384.

4 L. M. B. Zamilova, T. G. Biktimirova and V. I. Sokolova, Khim. Tekhnol. Topl. Masel, 1985, 12, 27.

5 J. S. F. Pereira, L. O. Diehl, F. A. Duarte, M. F. P. Santos, R. C. L. Guimarães, V. L. Dressler and E. M. M. Flores, J. Chromatogr. A, 2008, 1213, 249.

6 P. A. Mello, C. K. Giesbrecht, M. S. Alencar, E. M. Moreira, J. N. G. Paniz, V. L. Dressler and E. M. M. Flores, Anal. Lett., 2008, 41, 1623.

7 P. A. Mello, J. S. F. Pereira, D. P. Moraes, V. L. Dressler, E. M. M. Flores and G. Knapp, J. Anal. At. Spectrom., 2009, 24, 911.

8 F. G. Santos, A. C. Pereira, S. M. Cruz, C. A. Bizzi, E. M. M. Flores and B. F. Reis, Anal. Methods, 2015, 7, 4769. 9 L. Silverman, Ind. Eng. Chem., Anal. Ed., 1941, 13, 524.

10 J. S. S. Oliveira, R. S. Picoloto, C. A. Bizzi, P. A. Mello, J. S. Barin and E. M. M. Flores, Talanta, 2015, 144, 1052.

11 J. Alvarado, M. Alvarez, A. R. Cristiano and L. Marcó, Fuel, 1990, 69, 128.

12 J. Zhang, L. Li, Q. Zhang, Y. Yang and Q. Jin, Pet. Sci. Technol., 2007, 25, 443.

13 A. Crawford, J. G. Palmer and H. H. Wood, Mikrochim. Acta, 1957, 2, 277. 
14 A. S. Camera, T. A. Maranhão, F. J. S. Oliveira, J. S. A. Silva and V. L. A. Frescura, J. Braz. Chem. Soc., 2015, 26, 2116.

15 P. Spectrometer, G. Yun-chuan, G. Qin-fen, S. Ming-xing, Z. Zhi-xiu and C. Zong-hong, Chin. J. Anal. Chem., 2007, 35, 1175.

16 M. Niemelä, S. M. Huttunen, S. S. Gornostayev and P. Perämäki, Microchim. Acta, 2009, 166, 255.

17 C. Bendicho, I. Lavilla, F. Pena-Pereira and V. Romero, J. Anal. At. Spectrom., 2012, 27, 1831.

18 M. Wasilewska, W. Goessler, M. Zischka, B. Maichin and G. Knapp, J. Anal. At. Spectrom., 2002, 17, 1121.

19 J. S. F. Pereira, L. S. F. Pereira, P. A. Mello, R. C. L. Guimarães, R. A. Guarnieri, T. C. O. Fonseca and E. M. M. Flores, Anal. Chim. Acta, 2014, 844, 8.

20 L. S. F. Pereira, M. F. Pedrotti, T. M. Miceli, J. S. F. Pereira and E. M. M. Flores, Talanta, 2016, 152, 371.

21 C. Duyck, N. Miekeley, T. C. O. Fonseca, P. Szatmari and E. V. S. Neto, J. Braz. Chem. Soc., 2008, 19, 978.

22 L. Perna, F. Bocci, L. A. Heras, J. Pablo and M. Betti, J. Anal. At. Spectrom., 2002, 17, 1166.
23 R. Nakada, Y. Takahashi, G. Zheng, Y. Yamamoto and H. Shimizu, Geochem. J., 2010, 44, 411.

24 A. Akinlua, N. Torto and T. R. Ajayi, Fuel, 2008, 87, 1469.

25 R. P. Gottikh, S. F. Vinokurov and B. I. Pisotskii, Dokl. Earth Sci., 2009, 425, 325.

26 T. Yasnygina, Y. M. Malykh, S. Rasskazov, S. P. Primina, T. Zemskaya and O. Khlystov, Dokl. Earth Sci., 2006, 411, 1237.

27 L. Zhang, Y. Zhao, Z. Jin, G. Bai and L. Yang, Energy Explor. Exploit., 2009, 27, 47.

28 K. T. Akeda and Y. B. K. Rikawa, Jpn. Anal., 2005, 54, 939.

29 M. E. Kitto, D. L. Anderson, G. E. Gordon and I. Olmez, Environ. Sci. Technol., 1992, 26, 1368.

30 J. S. A. Silva, T. A. Maranhão, F. J. S. d. Oliveira, A. J. Curtius and V. L. A. Frescura, J. Braz. Chem. Soc., 2014, 25, 1062.

31 P. Kulkarni, S. Chellam and M. P. Fraser, Atmos. Environ., 2006, 40, 508.

32 T. G. Dzubay, R. K. Stevens, G. E. Gordon, I. Olmez, A. E. Sheffleld and W. Courtney, J. Environ. Sci. Technol., 1988, 22, 46. 